\title{
Influence of MWCNTs on $\beta$-Phase PVDF and Triboelectric Properties
}

\author{
Sejung Kim, ${ }^{1,2}$ Youngjun Song, ${ }^{2}$ and Michael J. Heller ${ }^{3}$ \\ ${ }^{1}$ Materials Science and Engineering, University of California San Diego, La Jolla, CA 92093, USA \\ ${ }^{2}$ Department of Electrical and Computer Engineering, University of California San Diego, La Jolla, CA 92093, USA \\ ${ }^{3}$ Department of Nanoengineering, University of California San Diego, La Jolla, CA 92093, USA
}

Correspondence should be addressed to Michael J. Heller; mheller@ucsd.edu

Received 20 March 2017; Accepted 7 May 2017; Published 16 July 2017

Academic Editor: Victor M. Castaño

Copyright (C) 2017 Sejung Kim et al. This is an open access article distributed under the Creative Commons Attribution License, which permits unrestricted use, distribution, and reproduction in any medium, provided the original work is properly cited.

\begin{abstract}
The surface of multiwalled carbon nanotubes (MWCNTs) was chemically modified using 1-pyrenebutyric acid (PBA) to improve its compatibility with polyvinylidene fluoride (PVDF). The carboxylic acid groups of the MWCNTs-PBA (PCNTs) provide a $\beta$ phase nucleation site to the fluorine of PVDF along their surface. The content of the $\beta$-phase crystalline structure of PVDF was found to be the highest at a concentration of $1.0 \mathrm{wt} . \%$ of PCNTs, and these PVDF-PCNTs composites were utilized as active layers in triboelectric devices. The maximum output voltage achieved was 16 volts at a concentration of 1.0 wt. $\%$ of PCNTs in the PVDF composites.
\end{abstract}

\section{Introduction}

Mechanical energy harvesting technology has been investigated in lightweight and biocompatible devices as a wireless power source by employing piezoelectric materials [1-3]. Among all of the available piezoelectric materials, polyvinylidene (PVDF) and its derivatives have been singled out in flexible nanogenerators due to their high dielectric, piezoelectric, and ferroelectric properties [4-6]. The $\beta$-phase of PVDF with the all-trans conformation of fluorine atoms leads to high values of electric polarization to the unit cell $[7,8]$. Generally, the $\beta$-phase of the crystalline structure of PVDF can be enhanced by either mechanical deformation in the uniaxial or biaxial directions or application of a highly intense electric field to the PVDF film above its glass transition temperature [9-11]. As a new energy harvesting technology, contact-electrification based energy conversion devices, that is, triboelectric nanogenerators, have been developed in such a way that they can achieve high efficiency for producing large output power as well as easy fabrication [12-14]. In addition, triboelectric performance can be enhanced by regulating the surface morphology. Regarding the PVDF composites, multiwalled carbon nanotubes (MWCNTs) in PVDF composites can produce inductive charges on the surface during electrospinning, wherein the $\beta$-phase of PVDF crystallizes on the surface of the MWCNTs, resulting in the transformation of local amorphous regions into the $\beta$-phase crystalline structures $[11,15,16]$.

In the present work, we investigate the triboelectric performance of PVDF composites with MWCNTs, corresponding to the change in the content of $\beta$-phase crystalline structure. Also, the surface of the MWCNTs is chemically modified with 1-pyrenebutyric acid (PBA) (PCNTs) in order to investigate the effect of transformation of $\beta$-phase crystalline structures of PVDF and their morphological features with respect to the amount of PCNTs loaded in PVDF.

\section{Experiments}

Synthesis of Nanocomposite Particles. The MWCNTs were first dispersed in $\mathrm{N}$-methyl-2-pyrrolidone (NMP) in an ultrasonication bath for $3 \mathrm{hrs}$. Then, $10 \mathrm{mM}$ of 1-pyrenebutyric acid (PBA) was added to the MWCNTs (Cheap Tubes, Inc.) suspension and the mixture was magnetically stirred for 
3 hrs. Once the reaction was complete, the mixture was washed with ethanol at least 5 times, and the resulting PBA-MWCNTs (PCNTs) particles were dispersed in $N, N$ dimethylformamide (DMF) for the next composite reaction. PVDF $\left(M w=50,000 \mathrm{~kg} \mathrm{~mol}^{-1}\right)$ powder was purchased from Sigma-Aldrich and used as received. PVDF was dissolved in $\mathrm{DMF}$ at a concentration of $100 \mathrm{mg} / \mathrm{mL}$ at room temperature in order to prevent crystallization during the dissolving process. Then, the chemically synthesized nanoparticle composite was added to the PVDF solution at different ratios by weight. The mixture was treated in an ultrasonication bath for 30 minutes yielding a uniformly mixed PCNTs-PVDF suspension. To obtain the PVDF based nanocomposites, the suspension was dropped onto a plain glass substrate at $200 \mu \mathrm{L} / \mathrm{in}^{2}$, and the DMF was evaporated by drying in an oven at $85^{\circ} \mathrm{C}$ overnight.

Fabrication of Triboelectric Device and Performance Test. PVDF based nanocomposite nanogenerator devices were fabricated by constructing a sandwiched structure. The thin film of the PVDF nanocomposite is attached to a Cu tape that acts as an electrode and is protected from mechanical damage by a Kapton film. The second layer is a piece of aluminum tape on a Kapton film as well. The two substrates were connected through an acryl spacer with an adhesive on both sides, maintaining a gap $(1 \mathrm{~mm})$ between the substrates. In order to evaluate the performance of the device, open-circuit voltages of PVDF composite films are measured with an oscilloscope (Tektronix, TDS 2014B) under a periodical compressive force of about $30 \mathrm{~N}$, vertically applied at a frequency of $3 \mathrm{~Hz}$. Noise reduction of the collected voltage variations of the PVDF nanocomposites was accomplished using a band-pass filter computer program.

Characterization. The morphologies of the as-prepared composites were observed by scanning electron microscopy (ESEM, Hitachi) at an acceleration voltage of $10 \mathrm{kV}$ and atomic force microscopy (AFM, Veeco model). X-ray diffraction (XRD) was carried out with the 2-theta range from 10 to $60^{\circ}$ at a scan rate of $0.02^{\circ} \mathrm{min}^{-1}$ using an XRD diffractometer (Rigaku Rotaflex) with $\mathrm{Cu} \mathrm{K} \alpha$ radiation. In addition, the crystal structure of the PVDF composite was investigated by Raman spectroscopy (Renishaw inVia Raman microscope). The modification of MWCNTs with PBA was confirmed by a Fourier transform infrared (FT-IR) spectrometer (PerkinElmer Spectrum Two).

\section{Results and Discussion}

A schematic representation shows the process for the chemical surface modification of MWCNTs and the triboelectric device structure shown in Figure 1. MWCNTs are chemically modified with $10 \mathrm{mM}$ of 1-pyrenebutyric acid (PBA) in $\mathrm{N}$-methyl-2-pyrrolidone (NMP) for 3 hours under gentle stirring. Subsequently, the chemically modified MWCNTs (PCNTs) are filtered under vacuum and washed with ethanol to remove the unreacted PBA followed by dispersion in $N, N$ dimethylformamide (DMF). The PVDF polymer solution is prepared at a concentration of $10 \mathrm{w} / \mathrm{v} \%$ in DMF by ultrasonication. In order to prepare the PCNTs-PVDF composite films, the prepared PVDF solution and PCNTs solution are mixed by ultrasonication for 1 hour with varying weight ratios of PCNTs from 0 to $2 \mathrm{wt} . \%$. Then, the PVDF-PCNTs solution was drop-casted $\left(200 \mu \mathrm{L} \mathrm{in}^{-2}\right)$ onto a plain glass substrate followed by evaporation of the solvent (DMF) in an oven at $85^{\circ} \mathrm{C}$ overnight under ambient conditions. These PVDF-PCNTs composite films are easily peeled off of the glass substrate without damage. Our schematic PVDF-PCNTs composites based triboelectric device is demonstrated in Figure 1(b). The first layer is composed of an aluminum tape on a Kapton film used as a substrate. The second layer is a piece of the polymer composite attached with a copper tape on a Kapton film as well. The two substrates are connected through an acryl spacer with adhesives on both sides, maintaining a gap $(1 \mathrm{~mm})$ between the substrates. In order to characterize the electric output, open-circuit voltages $\left(V_{\mathrm{oc}}\right)$ of PVDF-PCNTs based triboelectric devices fabricated by different types of PVDF-PCNTs films were measured under a periodical compressive force of $30 \mathrm{~N}$ applied at a frequency of $3 \mathrm{~Hz}$.

The morphology of the PCNTs was investigated by a scanning electron microscope (SEM) in Figure S1a (in Supplementary Material available online at https:/doi.org/ $10.1155 / 2017 / 2697382$ ), which showed entangled individual features on the substrate. The morphological features of the PVDF composite films were analyzed by using SEM (Figure 2) for pure PVDF, PVDF-PCNTs, and PVDF-MWCNTs composite films. While the pure PVDF has a large grain boundary domain $(\sim 5 \mu \mathrm{m})$ with corrugated surface, the PVDF-PCNTs film has a smaller grain boundary, and its surface is changed into an elongated corrugated surface after introducing the PCNTs into the PVDF matrix (Figure 2(b)). In comparison, the nonmodified MWCNTs in PVDF films do not have a large effect on forming a grain boundary, but MWCNTs are aggregated in the middle of the PVDF films (Figure 2(c)). The cross-sectional SEM images of PVDF composite films also reveal how well dispersed the PCNTs (Figure 2(b)(iii)) and aggregates of MWCNTs are in the PVDF matrix (Figure 2(c)(iii)). Consequently, it is evident that the MWCNTs are uniformly dispersed in the whole PVDF matrix by increasing the affinity to the solvents and the polymer followed by preventing phase separation. In addition, roughness was investigated by atomic force microscopy (AFM) (Figure 3). The PVDF-PCNTs films were examined with the PCNTs concentrations of $0 \mathrm{wt} . \%$ and $1.0 \mathrm{wt} . \%$ to the PVDF. We denoted the PVDF composite film including 1.0 wt.\% PCNTs as PVDF-10PCNT. We examined RMS values at both large and small scales and found that the roughness does not change much between the two composites. The RMS values are 567.5 and $580.3 \mathrm{~nm}$ at $14.6 \times 14.6 \mu \mathrm{m}$ and 1.359 and $1.818 \mathrm{~nm}$ at $3 \times 3 \mu \mathrm{m}$ for the pure PVDF and PVDF-10PCNT, respectively. The roughness of PVDF films was not largely changed.

In order to analyze the crystalline structure of PVDFPCNTs composite films, X-ray diffraction (XRD) was carried out at the speed of $1^{\circ} / \mathrm{min}$ and the range from $10^{\circ}$ to $60^{\circ}$ (Figure 4(a)). This describes the XRD results for PVDFPCNTs composite films corresponding to the loaded amount 


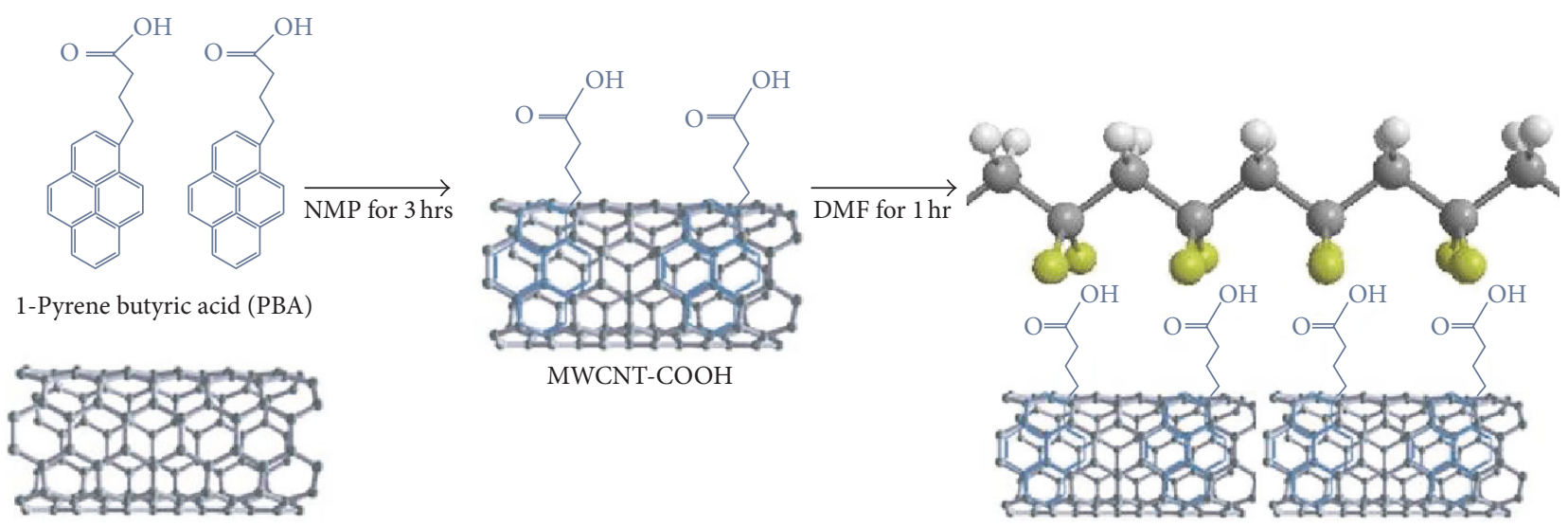

Multiwall carbon nanotubes

(a)

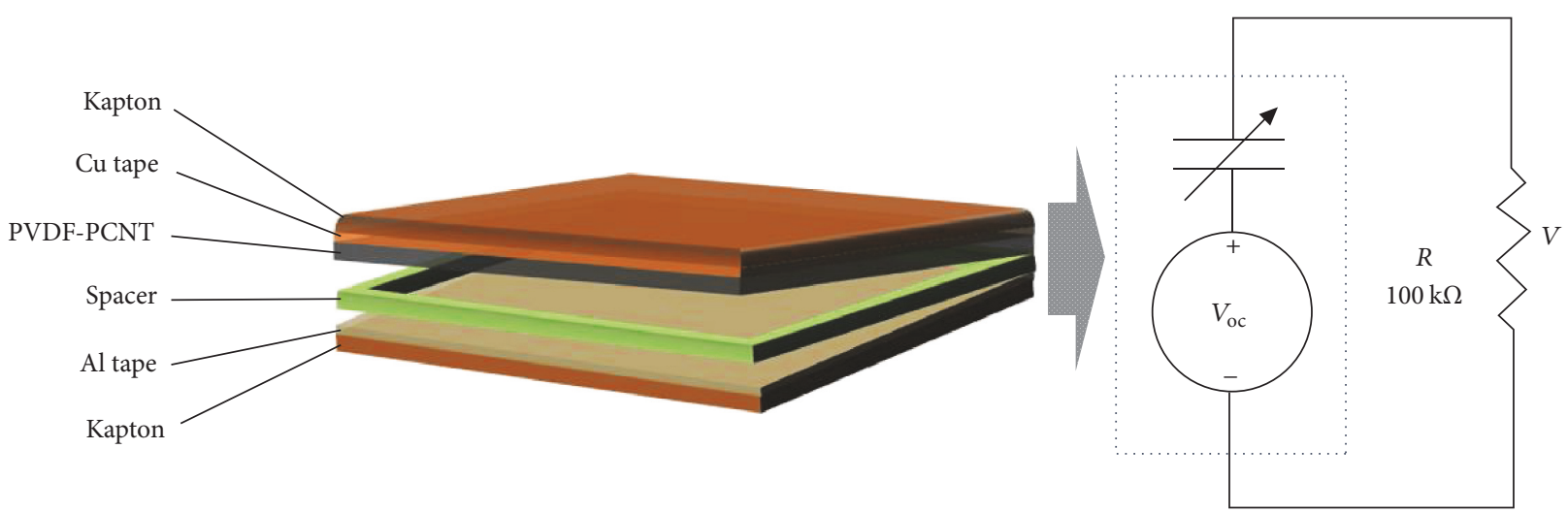

(b)

FIgURE 1: Schematic process to fabricate the nanocomposite of PVDF-PCNTs. (a) Surface modification of MWCNTs with PBA. (b) Nanocomposite of PVDF-PCNTs $(1 \times 1$ inch) and circuit diagram of the PVDF composite-based triboelectric device.

of PCNTs. In order to demonstrate the transition of PVDF crystalline structures, the pure PVDF and PVDF-MWCNTs composite films are also analyzed; it can be found that three distinctive peaks are observed at three different diffraction angles $(2 \theta): 18.4^{\circ}, 19.9^{\circ}$, and $26.5^{\circ}$ corresponding to (020), (110), and (021) crystal plane from the $\alpha$-phase crystalline structure [8]. When it comes to the PVDF-PCNTs composite films, the peak at $2 \theta=20.8^{\circ}$ is observed corresponding to (110) of the $\beta$-phase crystalline structure, which indicates that the crystalline phase transition occurred from the $\alpha$-phase to the $\beta$-phase. According to the amount of PCNTs in the PVDF films, the nanocomposite with $1.0 \mathrm{wt} . \%$ PCNTs loading can be observed to possess the highest relative peak intensity at 20.8 when the $\beta$-phase is increased. This indicates that the addition of PCNTs can induce phase transition from the $\alpha$-phase crystalline structure to the $\beta$-phase crystalline structure in the PVDF-PCNTs nanocomposite films. In addition, the PVDF-PCNTs nanocomposite film with PCNT $1.0 \mathrm{wt}$ \% loading can also be confirmed to possess the highest relative peak intensity at $=20.6^{\circ}$ and $\beta$-phase crystalline structure decreased upon a further increase of the PCNTs concentration to $2 \mathrm{wt} . \%$.
For the further investigation of crystalline transition of PVDF-PCNTs nanocomposite films, FT-IR was carried out in the region between 1500 and $550 \mathrm{~cm}^{-1}$ with a spectral resolution of $1 \mathrm{~cm}^{-1}$. For each spectrum, 10 scans were averaged. Figure 4(b) shows FT-IR spectra for nanocomposite films of the pure PVDF and PVDF with different PCNTs concentrations. As demonstrated in the XRD results, it was found that the PVDF-PCNTs nanocomposite films consisted of the $\alpha$-phase and the $\beta$-phase PVDF. The absorbance bands at $613 \mathrm{~cm}^{-1}$ ( $\mathrm{CF}_{2}$ bending), $763 \mathrm{~cm}^{-1}\left(\mathrm{CF}_{2}\right.$ wagging vibration), and $975 \mathrm{~cm}^{-1}\left(\mathrm{CF}_{2}\right.$ rocking vibration) were related to the $\alpha$-phase [8]. However, the absorbance bands at $838 \mathrm{~cm}^{-1}$ $\left(\mathrm{CH}_{2}\right.$ rocking), $875 \mathrm{~cm}^{-1}\left(\mathrm{CF}_{2}\right.$ stretching), $1173 \mathrm{~cm}^{-1}\left(\mathrm{CH}_{2}\right.$ wagging), and $1231 \mathrm{~cm}^{-1}\left(\mathrm{CH}_{2}\right.$ rocking) were observed, which were related to the $\beta$-phase PVDF [11]. From Figure 4(b), it was found that the $\alpha$-phase crystalline structure certainly exists in the PVDF-PCNTs nanocomposite films with various PCNT loadings by inducing the nucleation of the $\beta$-phase. We believe that the crystalline phase transition from $\alpha$ - to $\beta$-phase is due to the fact that the carboxylic acid groups enhance the compatibility of the PCNTs with $\mathrm{CF}_{2}$ groups in the PVDF due to strong interaction that can induce the 


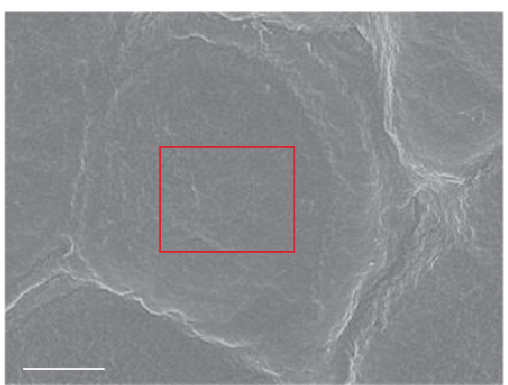

(i)

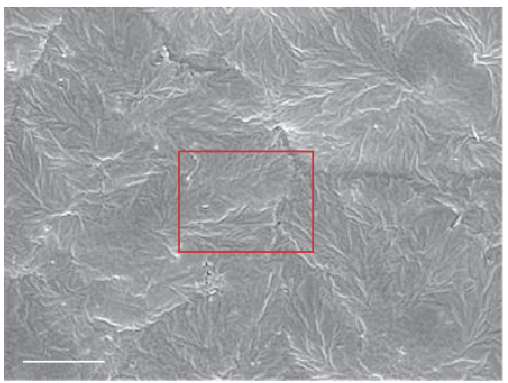

(i)

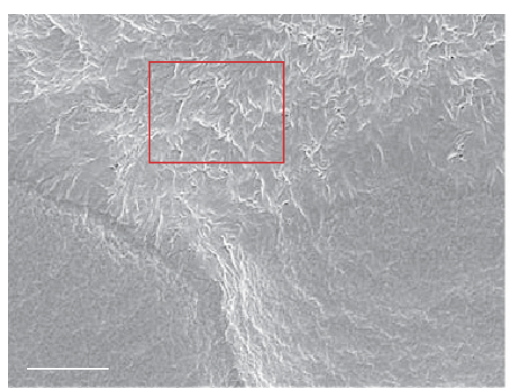

(i)

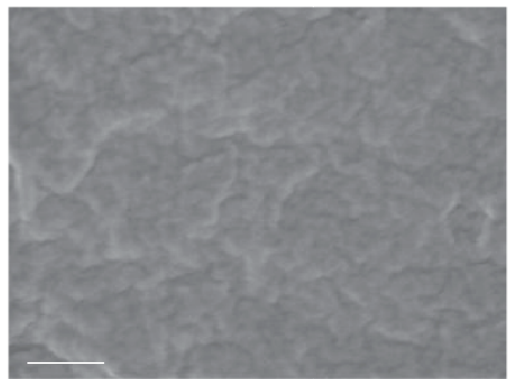

(ii)

(a)

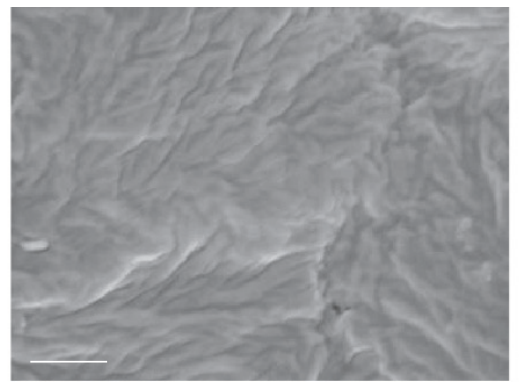

(ii)

(b)

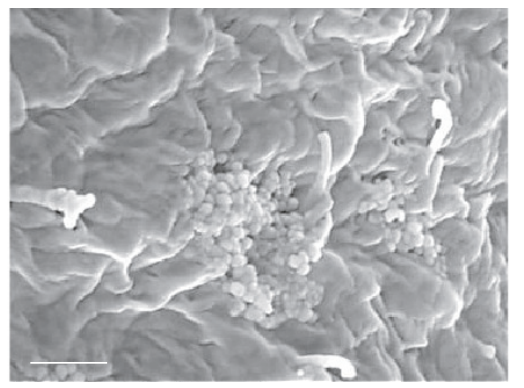

(ii)

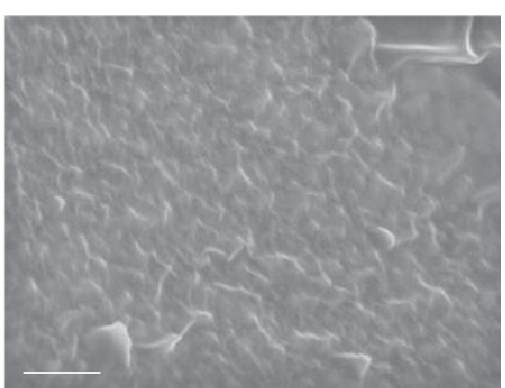

(iii)

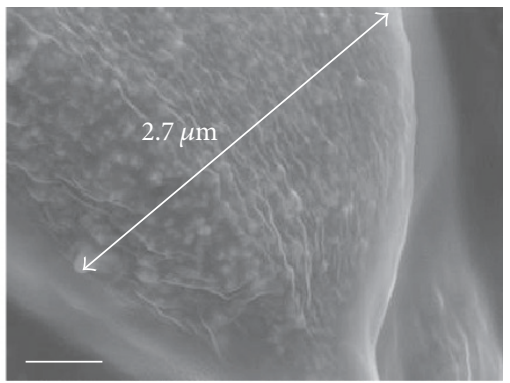

(iii)

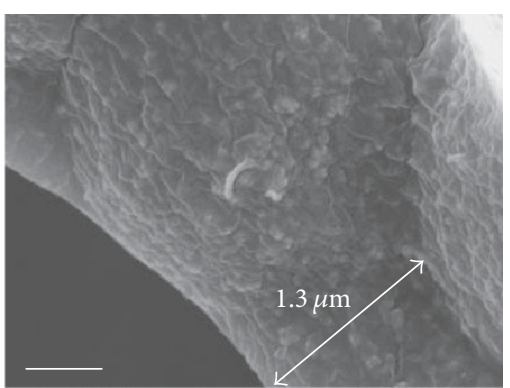

(iii)

(c)

Figure 2: Surface characterization of PVDF-PCNTs nanocomposite films using SEM. (a) Pure PVDF, (b) PVDF-10PCNT, and (c) PVDFMWCNTs composite films. (i) Top-view SEM images of each composite film and (ii) magnification of the rectangular region. (iii) Crosssectional SEM images of each composite film. Scale bar is $2 \mu \mathrm{m}$ for (a)-(i), (b)-(i), and (c)-(i) and $500 \mathrm{~nm}$ for the rest of the images.

crystallization of $\beta$-phase PVDF along the surface-mediated MWCNTs $[15,17,18]$. Nonetheless, the absorbance bands from the $\beta$-phase increase significantly at four different bands, while the peaks corresponding to the $\alpha$-phase crystal decrease. Therefore, the FT-IR spectra results indicate that $\alpha$-phase crystal peaks were transformed to $\beta$-phase crystal peaks by introducing the nucleation site from PCNTs, which is consistent with the results of XRD analysis.

Figure 5(a) shows the open-circuit voltage output of the five different PVDF-PCNTs types: pure PVDF and PVDFPCNTs with four different loading amounts of PCNTs. While the mechanical deformation is applied at $3 \mathrm{~Hz}$, the response time is 1.17 and $0.93 \mathrm{sec}$ for the pure PVDF and PVDF10PCNT composite films, respectively (Figure S2). The results clearly show that the order of the output performance of each of the PVDF-PCNTs compositions is PVDF < PVDF-20PCNTs < PVDF-01PCNTs < PVDF-05PCNTs <
PVDF-10PCNT. The maximum output voltage is up to 16 volts, which is almost 8 times as high as the pure PVDF based triboelectric devices. Therefore, we assumed that the increased $\beta$-phase content is attributed to the enhancement of the triboelectric effect due to its polarizability of the crystalline structure of PVDF.

As a result of the periodic mechanical deformation between the PVDF-PCNTs films and the electrode, electrostatic charges are oppositely generated and distributed on each surface of the insulating polymeric materials. The produced charges will not be conducted away or neutralized due to the insulating nature of polymer films, resulting in the accumulation of charges on the surface of composite films. Then, the charges will be conducted across the external load between the two electrodes to minimize the energy created by the triboelectric potential. Simultaneously, mechanical compression between the two layers of polymers leads to a 


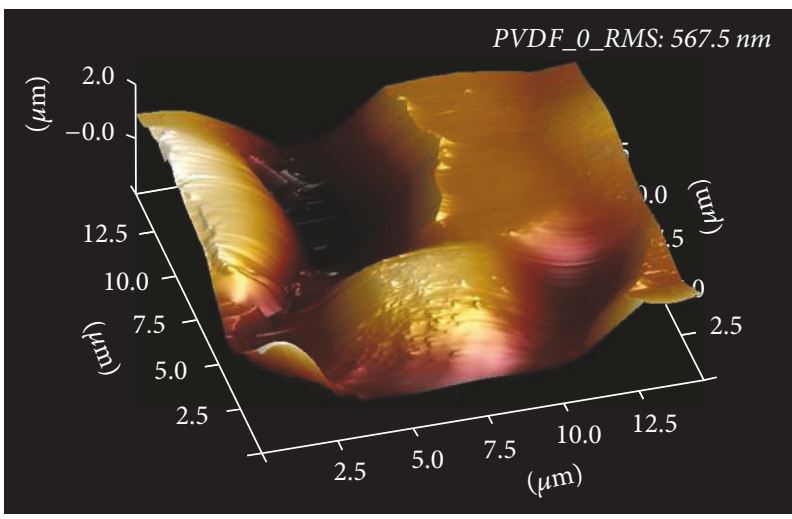

(a)

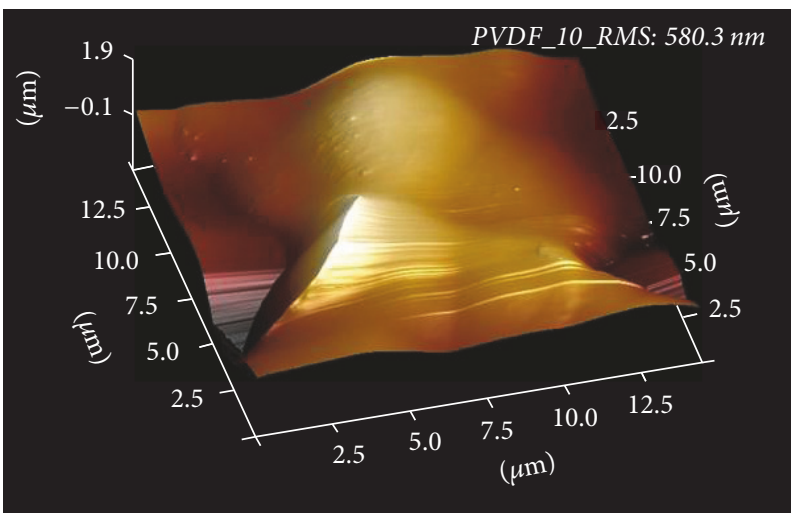

(c)

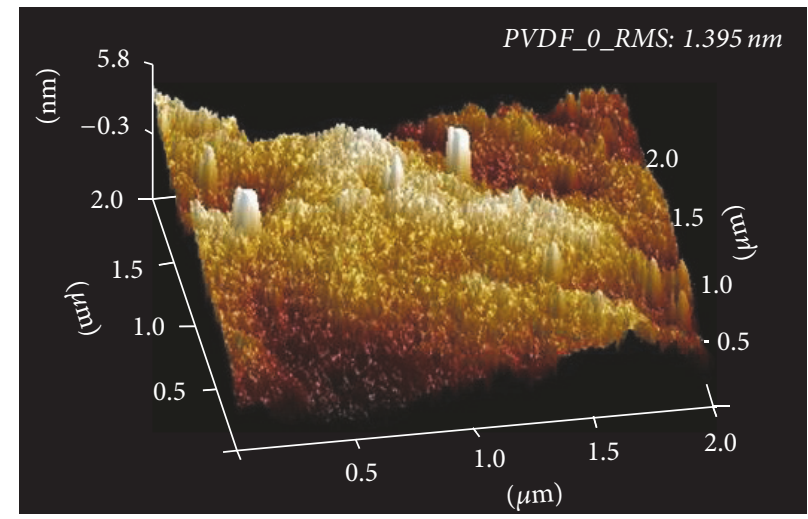

(b)

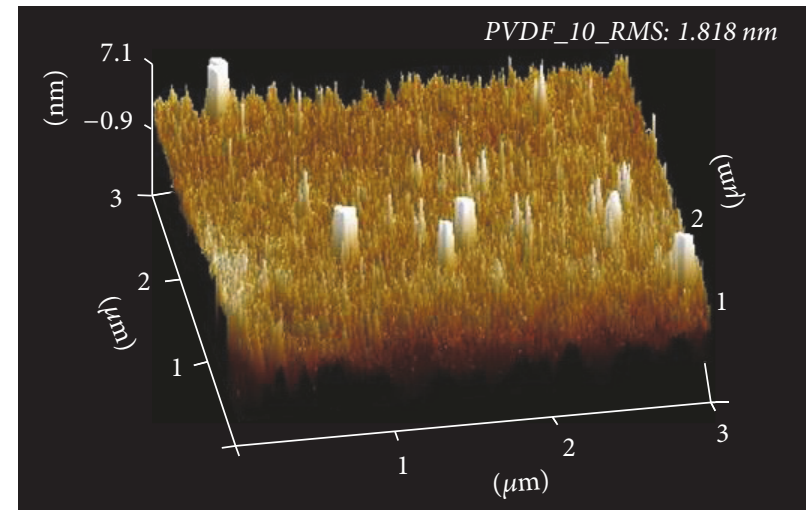

(d)

FIGURE 3: Determination of roughness of PVDF composite films by AFM analysis. $(a, b)$ The pure PVDF surface AFM images at a scale of $14.6 \mu \mathrm{m} \times 14.6 \mu \mathrm{m}$ and $2 \mu \mathrm{m} \times 2 \mu \mathrm{m}$, respectively. (c, d) PVDF-10PCNT surface images at a scale of $14.6 \mu \mathrm{m} \times 14.6 \mu \mathrm{m}$ and $3 \mu \mathrm{m} \times 3 \mu \mathrm{m}$, respectively.

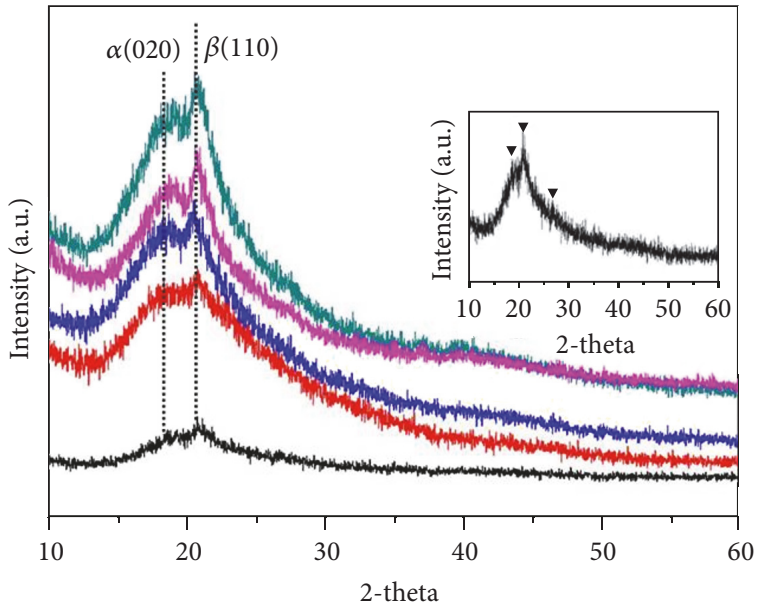

$\begin{array}{ll}- & \text { PVDF } \\ - & \text { PVDF01PCNT } \\ - & \text { PVDF05PCNT }\end{array}$

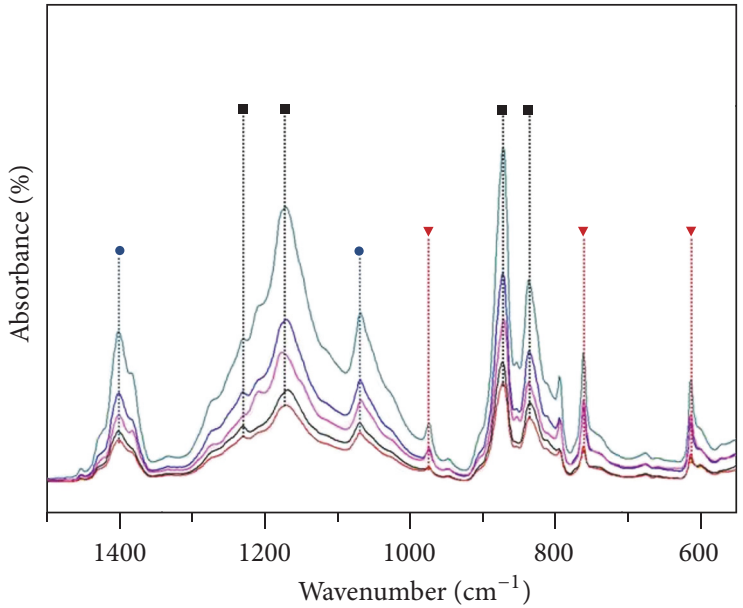

PVDF
- PVDF01PCNT
- PVDF05PCNT

- PVDF20PCNT

- $\beta$-phase

$\checkmark \alpha$-phase

- co-band

FIGURE 4: Spectroscopic analysis for the transition of crystalline structure of PVDF composite films. (a) XRD patterns and (b) FT-IR spectra of the pure PVDF and PVDF-PCNTs nanocomposite films corresponding to the amount of PCNTs from 0 to 2 wt.\%. 


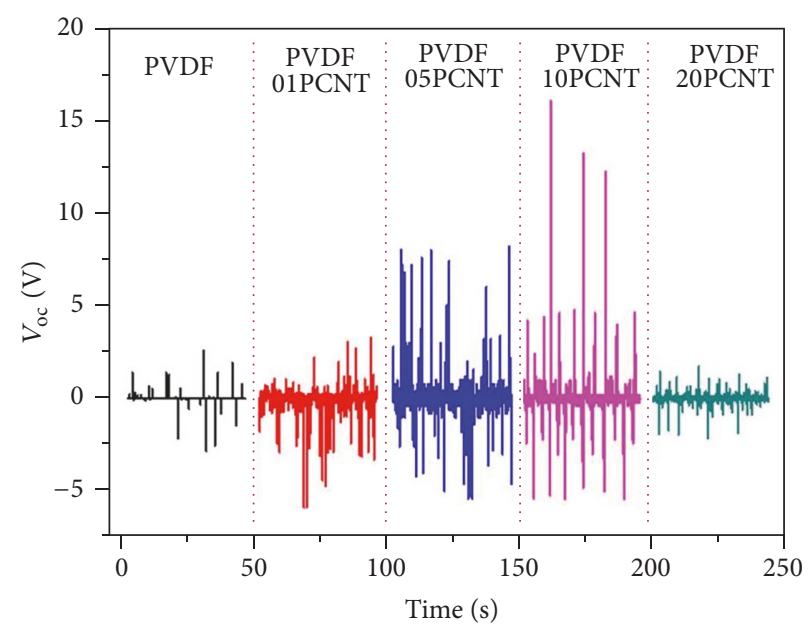

(a)

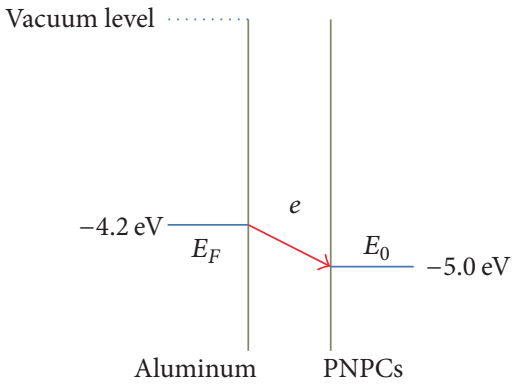

(b)

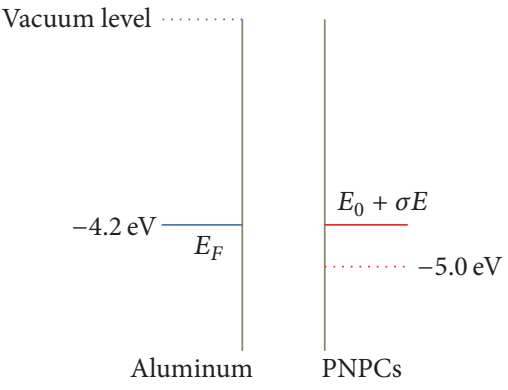

(c)

Figure 5: (a) Triboelectric device of $V_{\text {oc }}$ with different loading PCNTs from 0 to 2 wt.\%. (b, c) The mechanism of the band energy alignment for electron transport in the triboelectric device during mechanical deformation.

reduction of the interplanar distance. According to the literature, we need to consider the relationship between the surface morphology and the triboelectric effect in order to enhance the triboelectric device performance because the surface triboelectric charges are the direct driving force for transport of induced electrons between electrodes in a triboelectric device $[12-14,19]$. Firstly, the change in the capacitance is largely improved during the mechanical deformation due to the presence of the air void (low dielectric constant, $\sim 1$ ). Secondly, the triboelectric effect with a structured surface is largely enhanced due to the increased interface with the air corresponding to the unstructured surface followed by generating more surface charges during the friction. Lastly, the triboelectric charges are more easily separated due to their fast charge saturation on the structured surface. However, in our device using PVDF-PCNTs nanocomposite films, the roughness is not largely changed compared to the pure PVDF films.

Lastly, we now consider the charge transfer between the PVDF-PCNTs nanocomposite dielectric layers and electrodes. In our device, the unequal effective work function of two electrodes was adopted such as $\mathrm{Al}$ and $\mathrm{Cu}$. Generally, the electrons will flow from the lower work function of materials into the higher work function of materials. As illustrated in Figures 5(b) and 5(c), we adopted the two different work function electrodes, aluminum $(4.2 \mathrm{eV})$ and copper $(4.7 \mathrm{eV})$. Moreover, PVDF has an energy level with an effective work function of around $5.0 \mathrm{eV}$. When it comes to the charge transfer, electrons flow from the Fermi level of aluminum to the PVDF due to its lower work function during the friction followed by making the PVDF surface negatively charged. Then, the electric field is developed, followed by the decrease of the potential difference between aluminum and PVDF, and the charge transfer will stop until the saturation of electrons. The center of the positive charges does not coincide in every molecule of PVDF in its polar $\beta$-phase, but the bond charge is zero when $\beta$ forms naturally because of the random arrangement of dipoles. As a result, an enhanced charge density of triboelectric effect can be expected. The surface charge density thus determines the amount of induced electrons that flow to screen electric field from the triboelectric charges.

\section{Conclusion}

In conclusion, we have demonstrated the relationship between the dielectric nanocomposites and the triboelectric effect by adopting the PVDF based nanocomposites composed of PVDF-PCNTs to open a novel and simple approach. The PVDF-PCNTs films play an important role and benefit the performance aspect and should be considered with charge generation, charge separation, and charge transport in the device. PVDF-PCNTs are a soft material and could easily be fabricated into a patterned morphology by simple solvent 
evaporation. The increase in $\beta$-phase of PVDF-PCNTs nanocomposite films was seen in terms of the addition of nucleation sites without phase separation in the PVDF matrix. Lastly, the different energy level of materials is helpful for charge separation/transfer in the device.

\section{Conflicts of Interest}

The authors declare that there are no conflicts of interest regarding the publication of this paper.

\section{References}

[1] Z. L. Wang and J. Song, "Piezoelectric nanogenerators based on zinc oxide nanowire arrays," Science, vol. 312, no. 5771, pp. 243246, 2006.

[2] S.-H. Shin, Y.-H. Kim, M. H. Lee, J.-Y. Jung, and J. Nah, "Hemispherically aggregated $\mathrm{BaTiO} 3$ nanoparticle composite thin film for high-performance flexible piezoelectric nanogenerator," ACS Nano, vol. 8, no. 3, pp. 2766-2773, 2014.

[3] K.-I. Park, C. K. Jeong, J. Ryu, G.-T. Hwang, and K. J. Lee, “Flexible and large-area nanocomposite generators based on lead zirconate titanate particles and carbon nanotubes," Advanced Energy Materials, vol. 3, no. 12, pp. 1539-1544, 2013.

[4] J. Wang, H. Li, J. Liu, Y. Duan, S. Jiang, and S. Yan, "On the $\alpha \rightarrow$ $\beta$ transition of carbon-coated highly oriented PVDF ultrathin film induced by melt recrystallization," Journal of the American Chemical Society, vol. 125, no. 6, pp. 1496-1497, 2003.

[5] L. Persano, C. Dagdeviren, Y. Su et al., "High performance piezoelectric devices based on aligned arrays of nanofibers of poly(vinylidenefluoride-co-trifluoroethylene)," Nature Communications, vol. 4, article 1633, 2013.

[6] M. Li, H. J. Wondergem, M.-J. Spijkman et al., "Revisiting the $\delta$-phase of poly(vinylidene fluoride) for solution-processed ferroelectric thin films," Nature Materials, vol. 12, no. 5, pp. 433438, 2013.

[7] E. Fukada, S. Tasaka, and H. Nalwa, Eds., Ferroelectric Polymer: Chemistry, Physics and Applications, Marcel Dekker, New York, NY, USA, 1995.

[8] S. Yu, W. Zheng, W. Yu, Y. Zhang, Q. Jiang, and Z. Zhao, "Formation mechanism of $\beta$-phase in PVDF/CNT composite prepared by the sonication method," Macromolecules, vol. 42, no. 22, pp. 8870-8874, 2009.

[9] P. Martins, J. S. Nunes, G. Hungerford et al., "Local variation of the dielectric properties of poly(vinylidene fluoride) during the $\alpha$ - to $\beta$-phase transformation," Physics Letters, Section A: General, Atomic and Solid State Physics, vol. 373, no. 2, pp. 177180, 2009.

[10] H. Tang, Y. Lin, and H. A. Sodano, "Enhanced energy storage in nanocomposite capacitors through aligned PZT nanowires by uniaxial strain assembly," Advanced Energy Materials, vol. 2, no. 4, pp. 469-476, 2012.

[11] Alamusi, J. Xue, L. Wu et al., "Evaluation of piezoelectric property of reduced graphene oxide (rGO)-poly(vinylidene fluoride) nanocomposites," Nanoscale, vol. 4, no. 22, pp. 7250-7255, 2012.

[12] F. R. Fan, L. Lin, and G. Zhu, "Kinetic competition model and size-dependent phase selection in 1-D nanostructures," Nano Letters, vol. 12, no. 6, pp. 3109-3114, 2012.

[13] L. Lin, Y. N. Xie, S. H. Wang et al., "Triboelectric active sensor array for self-powered static and dynamic pressure detection and tactile imaging," ACS Nano, vol. 7, no. 9, pp. 8266-8274, 2013.

[14] G. Zhu, Z.-H. Lin, Q. Jing et al., “Toward large-scale energy harvesting by a nanoparticle-enhanced triboelectric nanogenerator," Nano Letters, vol. 13, no. 2, pp. 847-853, 2013.

[15] G. H. Kim, S. M. Hong, and Y. Seo, "Piezoelectric properties of poly(vinylidene fluoride) and carbon nanotube blends: $\beta$-phase development," Physical Chemistry Chemical Physics, vol. 11, no. 44, pp. 10506-10512, 2009.

[16] P. Martins, C. Caparros, R. Gonçalves et al., "Role of nanoparticle surface charge on the nucleation of the electroactive $\beta$-poly(vinylidene fluoride) nanocomposites for sensor and actuator applications," Journal of Physical Chemistry C, vol. 116, no. 29, pp. 15790-15794, 2012.

[17] Z.-M. Dang, H.-Y. Wang, and H.-P. Xu, "Influence of silane coupling agent on morphology and dielectric property in BaTiO3/ polyvinylidene fluoride composites," Applied Physics Letters, vol. 89, no. 11, Article ID 112902, 2006.

[18] H. Yu, T. Huang, M. Lu, M. Mao, Q. Zhang, and H. Wang, "Enhanced power output of an electrospun PVDF/MWCNTsbased nanogenerator by tuning its conductivity," Nanotechnology, vol. 24, no. 40, Article ID 405401, 2013.

[19] S. Niu, S. Wang, L. Lin et al., "Theoretical study of contact-mode triboelectric nanogenerators as an effective power source," Energy and Environmental Science, vol. 6, no. 12, pp. 3576-3583, 2013. 

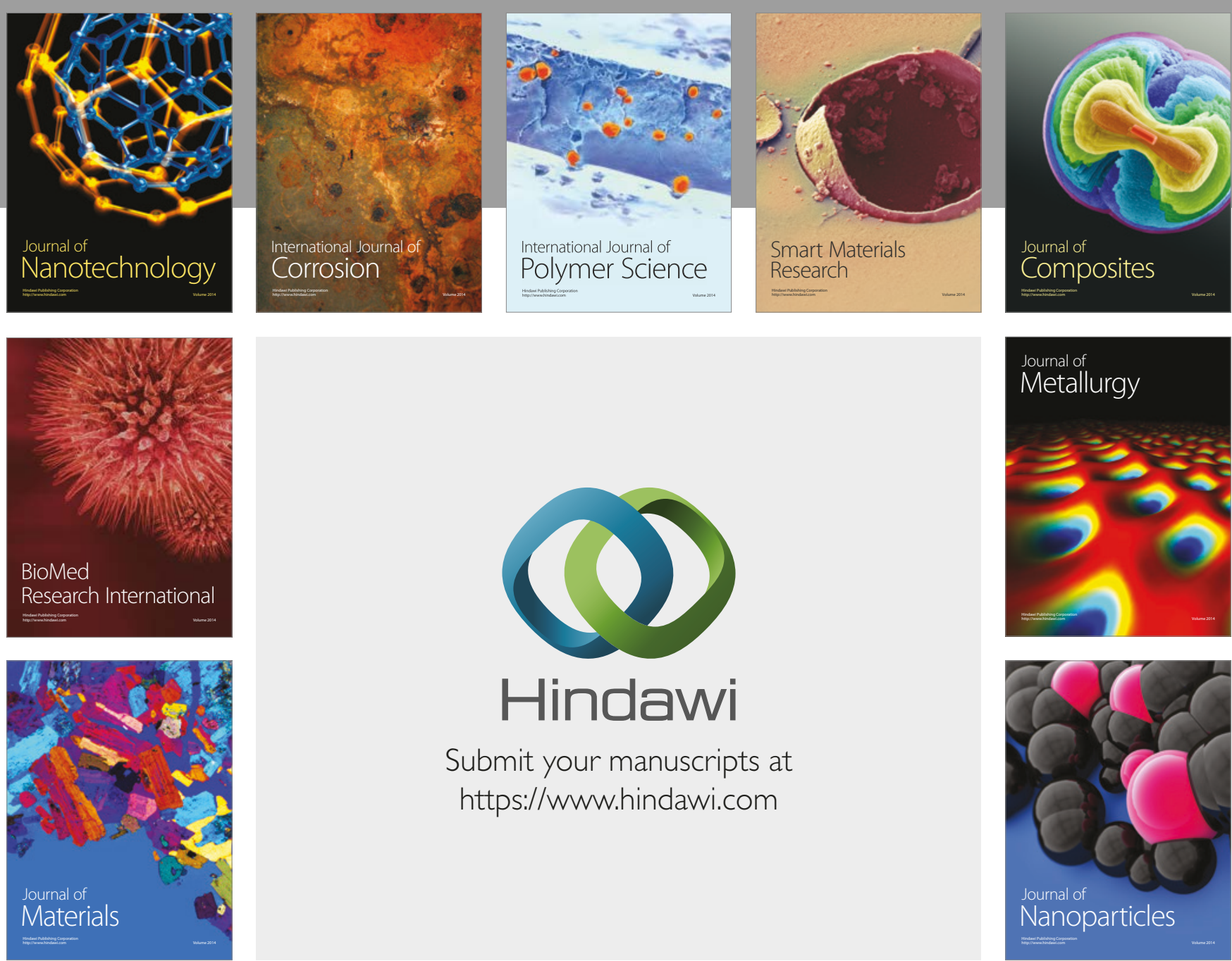

\section{Hindawi}

Submit your manuscripts at

https://www.hindawi.com
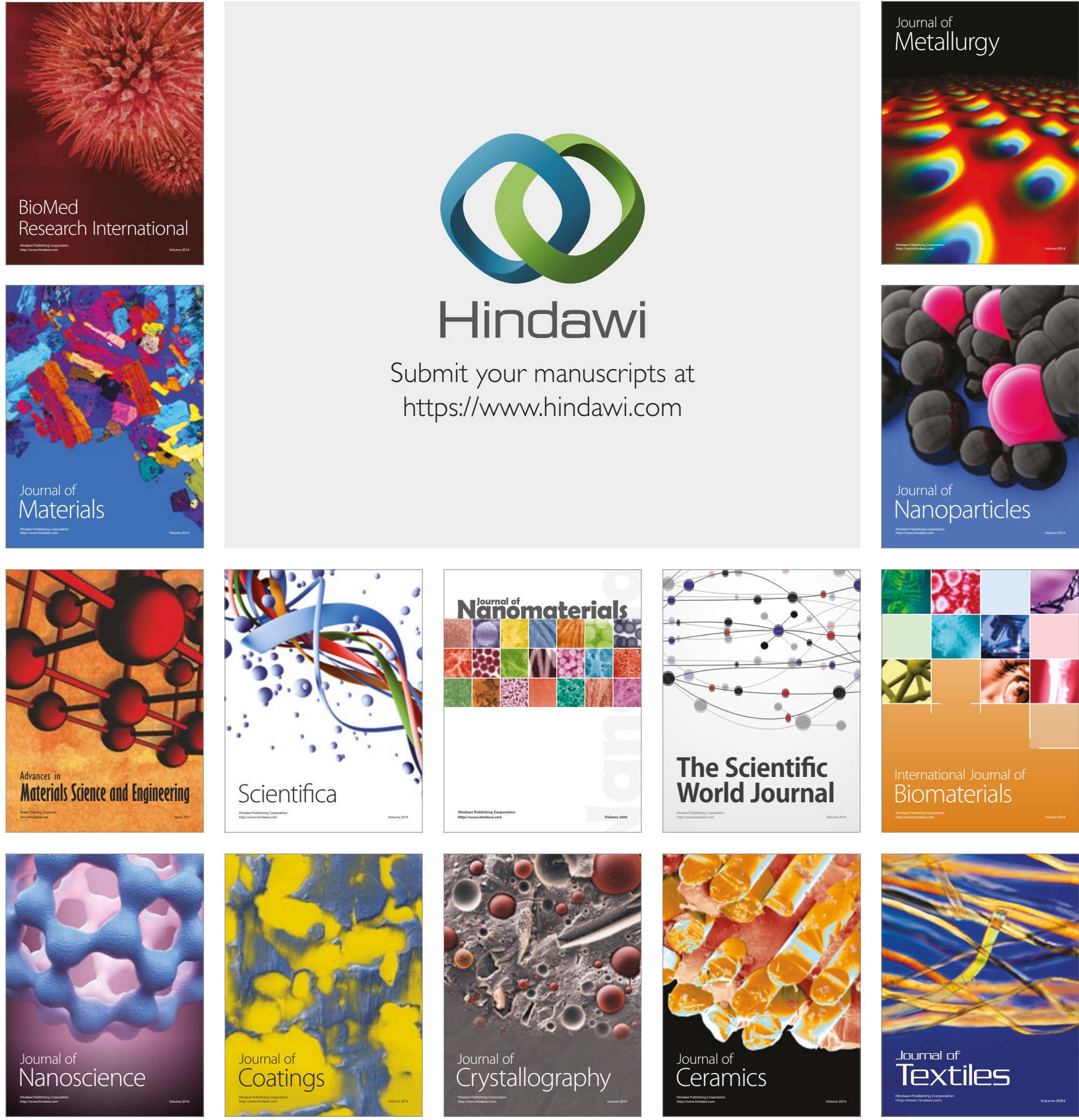

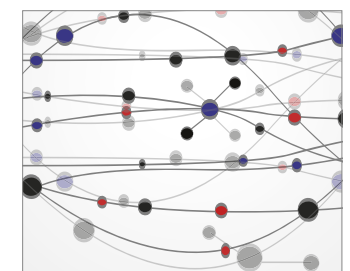

The Scientific World Journal
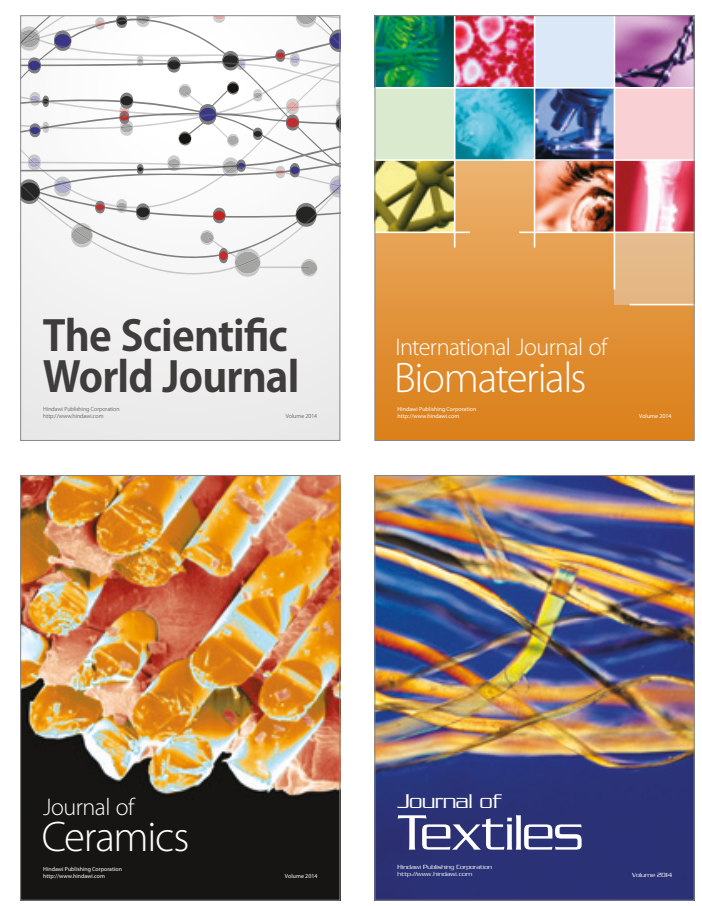